ROME1 prep. 98/1227

LPTHE - Orsay 98/63

ROME3 prep. $98 / 6$

\title{
Non-perturbatively Improved Heavy-Light Mesons: Masses and Decay Constants
}

\author{
D. Becirevic ${ }^{a}$, Ph. Boucaud ${ }^{a}$, J.P. Leroy ${ }^{a}$,

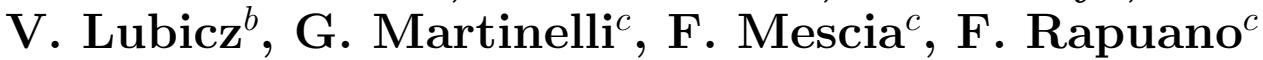 \\ a Laboratoire de Physique Théorique et Hautes Energies $\rrbracket$, \\ Université de Paris XI, Bâtiment 211, 91405 Orsay Cedex, France. \\ ${ }^{b}$ Dip. di Fisica, Univ. di Roma Tre and INFN, Sezione di Roma Tre, \\ Via della Vasca Navale 84, I-00146 Rome, Italy. \\ ${ }^{c}$ Dip. di Fisica, Univ. "La Sapienza" and INFN, Sezione di Roma, \\ P.le A. Moro, I-00185 Rome, Italy.
}

\begin{abstract}
We present a study of the heavy-light spectrum and of the $D$ - and $B$-meson decay constants. The results were obtained in the quenched approximation, by using the nonperturbatively improved Clover lattice action at $\beta=6.2$, with a sample of 100 configurations, on a $24^{3} \times 64$ lattice. After a careful analysis of the systematic errors present in the extraction of the physical results, by assuming quite conservative discretization errors, we find $f_{D_{s}}=231 \pm 12_{-1}^{+6} \mathrm{MeV}, f_{D}=211 \pm 14_{-12}^{+0} \mathrm{MeV}, f_{D_{s}} / f_{D}=1.10(2), f_{B_{s}}=204 \pm 16_{-0}^{+28} \mathrm{MeV}$, $f_{B}=179 \pm 18_{-9}^{+26} \mathrm{MeV}, f_{B_{s}} / f_{B}=1.14(3)_{-1}^{+0}$. Our results, which have smaller discretization errors than many previous estimates at fixed value of the lattice spacing $a$, support a large value of $f_{B}$ in the quenched approximation.
\end{abstract}

PACS numbers: 12.38.Gc,12.39.Hg,14.40.NdLb,13.25.Hw,13.30.Ce.

\footnotetext{
${ }^{1}$ Laboratoire associé au Centre National de la Recherche Scientifique - URA D00063.
} 


\section{Introduction}

In this paper, we present the results of a lattice calculation of physical quantities of phenomenological interest for heavy quarks, such as their mass spectrum and decay constants.

In order to reduce the systematic errors, we have performed calculations using the most recent developments in the lattice approach, namely:

1. The non-perturbatively improved lattice Clover action [1], which we denote as "Alpha action" [2, 35, 4] (see also [5]), with the coefficient of the chromomagnetic operator computed in Ref. [3];

2. Non-perturbatively improved vector and axial-vector currents, the renormalization coefficients of which have been computed, using the Ward Identities method [2, 6, 7], in Refs. [3, 田, 8];

The use of non-perturbatively improved actions and operators allows us to reduce the discretization errors to $\mathcal{O}\left(a^{2}\right)$. This is particularly important for heavy quark physics since, in current lattice simulations, the typical heavy quark mass $m_{Q}$ is rather large, $m_{Q} a \sim 0.3-0.6$.

Since the coefficient of the Clover term is known non-perturbatively, the hadron spectrum is definitively improved to $\mathcal{O}\left(a^{2}\right)$. Unfortunately, the program of removing all the $\mathcal{O}(a)$ corrections in the operator matrix elements out of the chiral limit has not been completed yet, although strategies to this purpose already exist [9, 10]. For this reason, in some cases, we have used the improvement coefficients $\left(b_{A}, c_{V}, b_{m}\right)$ evaluated at first order in (boosted) perturbation theory [11], thus leaving us with $\mathcal{O}\left(\alpha_{s}^{2} a m\right)$ corrections, where $m$ is the relevant quark mass.

After a careful analysis of the systematic uncertainties present in the extraction of the physical results, by assuming quite conservative errors, and bearing in mind the systematic effects due to the quenched approximation, the main results of our investigation are the following:

i) For $D$ mesons we find:

$$
\begin{aligned}
& f_{D}=211 \pm 14_{-12}^{+0} \mathrm{MeV}, \quad f_{D_{s}}=231 \pm 12_{-0}^{+6} \mathrm{MeV}, \quad\left(\frac{f_{D_{s}}}{f_{D}}\right)=1.10(2), \\
& f_{D^{*}}=245 \pm 20_{-2}^{+0} \mathrm{MeV}, \quad f_{D_{s}^{*}}=272 \pm 16_{-20}^{+0} \mathrm{MeV}, \quad\left(\frac{f_{D_{s}^{*}}}{f_{D^{*}}}\right)=1.11(3),
\end{aligned}
$$

where $f_{D^{*}}$ and $f_{D_{s}^{*}}$ are the vector-meson decay constants. The latter quantities are not measured experimentally, but enter the calculation of two-body non-leptonic $B$-decays computed using factorization [12]. Thus, they are useful for checking the factorization hypothesis with charmed vector mesons in the final states.

ii) For $B$ mesons, we find:

$$
\begin{array}{ccc}
f_{B}=179 \pm 18_{-9}^{+26} \mathrm{MeV}, & f_{B_{s}}=204 \pm 16_{-0}^{+28} \mathrm{MeV}, & \left(\frac{f_{B_{s}}}{f_{B}}\right)=1.14(3)_{-1}^{+0} \\
f_{B^{*}}=196 \pm 24_{-2}^{+31} \mathrm{MeV}, & f_{B_{s}^{*}}=229 \pm 20_{-16}^{+31} \mathrm{MeV}, & \left(\frac{f_{B_{s}^{*}}}{f_{B^{*}}}\right)=1.17(4)_{-3}^{+0}
\end{array}
$$


Following Ref. [13], we have also directly computed the ratio

$$
\frac{f_{B}}{f_{D_{s}}}=0.78 \pm 0.04_{-0}^{+11}
$$

from which, using the experimental result, $f_{D_{s}}^{(e x p .)}=254 \pm 31 \mathrm{MeV}$ [14], we find

$$
f_{B}=\frac{f_{B}}{f_{D_{s}}} \times f_{D_{s}}^{(\text {exp. })}=\left(198 \pm 24(\text { exp. })_{-10}^{+30}(\text { theo. })\right) \mathrm{MeV}
$$

Although with a larger error, the result in Eq. (田) is well compatible with the value given in (2).

iii) To reduce the effects of the quenched approximation, we have also used the Grinstein double-ratio method (illustrated below), obtaining

$$
r_{D}=\frac{f_{D_{s}}}{f_{D}}=1.19(5), \quad r_{B}=\frac{f_{B_{s}}}{f_{B}}=1.23(6), \quad \frac{f_{B}}{f_{D_{s}}}=0.71 \pm 0.04_{-0}^{+10}
$$

The latter ratio would give as the best estimate for $f_{B}$ :

$$
f_{B}=\left(180 \pm 26(\text { exp. })_{-10}^{+29}(\text { theo. })\right) \mathrm{MeV} .
$$

With the double ratio method we also obtained

$$
r_{B} / r_{D}=1.03(4)
$$

iv) We made a detailed study of the hyperfine splitting and of the scaling laws for masses and decay constants, as predicted by the heavy quark symmetry. The results of this study can be found below.

We now give the details of our analysis and of the methods used to extract the different physical quantities. Since most of the techniques are by now standard and have been described ad abundantiam in the literature? , we only focus on those points which are either less common or new. More details on the calibration of the lattice spacing and on the extraction of the hadron masses and matrix elements can be found in Refs. 17, 18].

The remainder of this paper is organized as follows. In Sec. 2, we list the main parameters of our simulation and introduce the basic notation necessary for the discussion of the results. The heavy-light meson masses and decay constants in lattice units are also given in this section. Since the systematic effects related to the extrapolation/interpolation to the physical point, although related, are quite different in the two cases, we present separately the physical predictions for $D$ and $B$ mesons, in Sec. 3 and Sec. 1 , respectively. In Sec. 5, we discuss the scaling laws predicted by the heavy quark effective theory (HQET) and other related subjects.

\footnotetext{
${ }^{1}$ This value has been recently updated by the same authors and reported to us by F. Parodi.

${ }^{2}$ Reviews, with complete lists of references, can be found in [15, 16].
} 


\section{Lattice results}

In this section, we give the essential information about our numerical calculation and establish the basic notation. We then present our results for the heavy-light meson masses and decay constants in lattice units.

The numerical simulation has been performed on a $24^{3} \times 64$ lattice, at $\beta=6.2$, in the quenched approximation. All results and errors have been obtained with a statistical sample of 100 independent gauge field configurations, using the jackknife method with different decimations. We have used the non-perturbatively improved lattice Clover action, with $c_{S W}=1.614$ [3]. We work with four values of $\kappa_{\text {light }}$, and four $\kappa_{\text {heavy }}$ :

- $0.1352\left(\kappa_{\ell_{1}}\right) ; 0.1349\left(\kappa_{\ell_{2}}\right) ; 0.1344\left(\kappa_{\ell_{3}}\right) ; 0.1333\left(\kappa_{\ell_{4}}\right)$,

- $0.1250\left(\kappa_{h_{1}}\right) ; 0.1220\left(\kappa_{h_{2}}\right) ; 0.1190\left(\kappa_{h_{3}}\right) ; 0.1150\left(\kappa_{h_{4}}\right)$.

From the study of the light-hadron spectrum, we obtained

- $a^{-1}\left(m_{K^{*}}\right)=2.75(17) \mathrm{GeV}$,

- $\kappa_{\text {crit }}=0.135845(25)$,

- $\kappa_{q}=0.135804(26)$,

$-\kappa_{s}=0.13482(12)$,

where $\kappa_{q}$, corresponds to the light quark mass $m_{q}$ (with $q=u, d$ ), and $\kappa_{s}$ to the strangequark mass, $m_{s}$. The above values have been obtained from the physical pion and kaon masses, by using the method of physical lattice planes [19]. All details regarding light hadron spectroscopy and decay constants, can be found in Refs. [17, 18].

For the mass spectrum, following the standard procedure, we measured suitable two-point correlation functions, from which we can isolate the lowest lying states

$$
C_{J J}(t)=\sum_{\vec{x}}\left\langle 0\left|J(\vec{x}, t) J^{\dagger}(0)\right| 0\right\rangle \stackrel{t \gg 0}{\longrightarrow} \frac{\mathcal{Z}_{J}}{\sinh \left(M_{J}\right)} e^{-M_{J} T / 2} \cosh \left[M_{J}\left(\frac{T}{2}-t\right)\right],
$$

where $J \equiv J_{P S}=\bar{Q} \gamma_{5} q$, or $J \equiv J_{V}^{k}=\bar{Q} \gamma^{k} q$. In Fig. 1, we show the effective masses for the pseudoscalar and vector heavy-light mesons at fixed heavy quark mass. By inspection, we established the fit intervals $t \in[20,28]$, and $t \in[22,28]$, for the pseudoscalar and vector cases, respectively. The resulting pseudoscalar and vector masses in lattice units, as well as the matrix elements, $\mathcal{Z}_{P S}=\left|\left\langle P S(\vec{p}=0)\left|J_{P S}\right| 0\right\rangle\right|^{2}$ and $\mathcal{Z}_{V}=\left|\left\langle V(\vec{p}=0 ; \lambda)\left|J_{V}^{k}\right| 0\right\rangle\right|^{2}$, are listed in Tab. 1. 

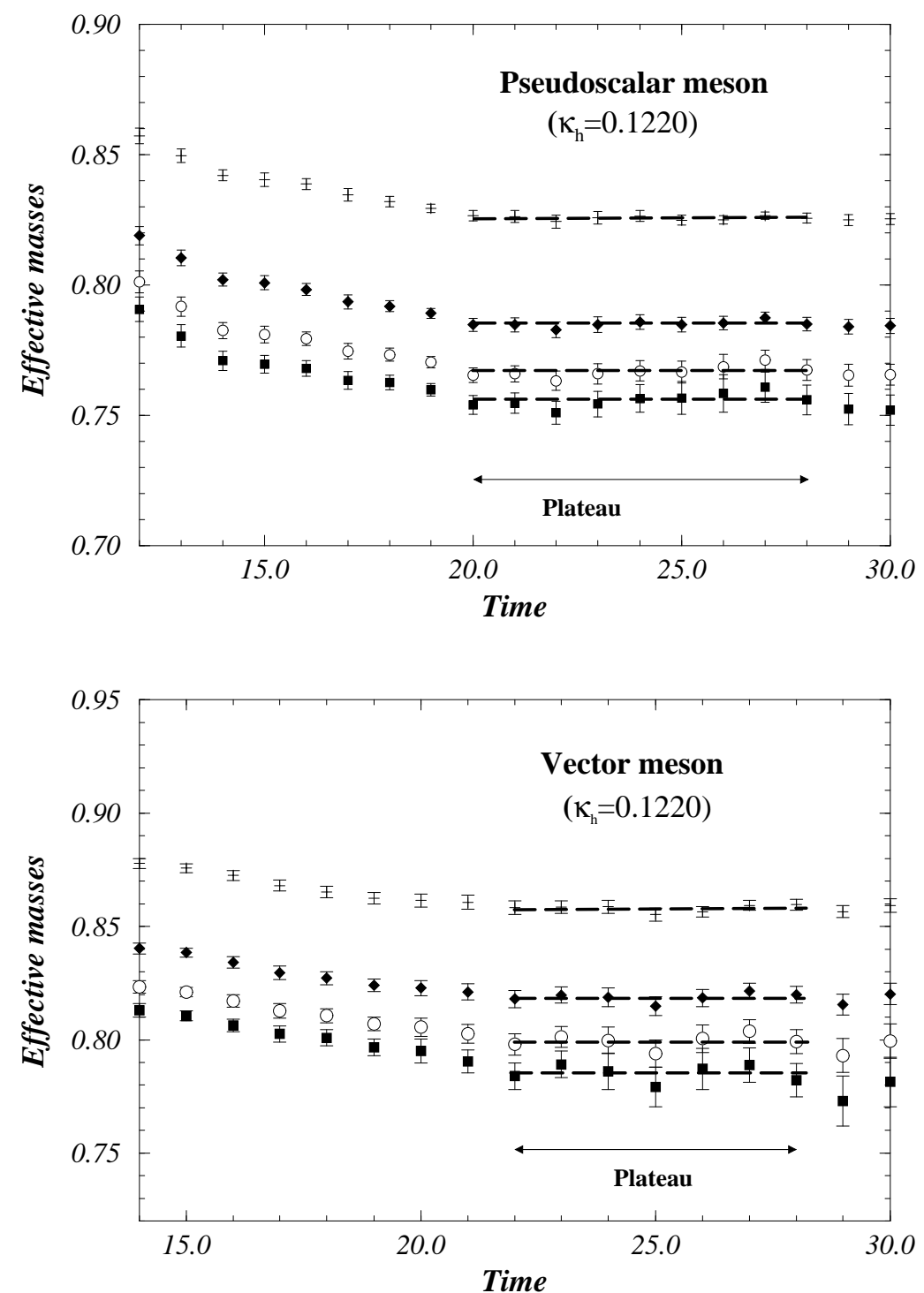

Figure 1: Effective masses of heavy-light pseudoscalar and vector mesons as a function of the time in lattice units. In each figure, the heavy quark mass (corresponding to $\kappa_{h}=0.1220$ ) is fixed, and combined with four different light quark masses. 


\begin{tabular}{|c|c|c|c|c|}
\hline "Flavor" content & $M_{P S}$ & $\mathcal{Z}_{P S}$ & $M_{V}$ & $\mathcal{Z}_{V}$ \\
\hline \hline$h_{4}-\ell_{4}$ & $1.0256(19)$ & $0.0254(9)$ & $1.0489(21)$ & $0.0120(6)$ \\
$h_{4}-\ell_{3}$ & $0.9868(29)$ & $0.0198(11)$ & $1.0104(33)$ & $0.0091(7)$ \\
$h_{4}-\ell_{2}$ & $0.9696(45)$ & $0.0176(16)$ & $0.9920(52)$ & $0.0077(9)$ \\
$h_{4}-\ell_{1}$ & $0.9584(67)$ & $0.0158(22)$ & $0.9783(77)$ & $0.0065(11)$ \\
\hline \hline$h_{3}-\ell_{4}$ & $0.9143(17)$ & $0.0237(8)$ & $0.9420(20)$ & $0.0105(5)$ \\
$h_{3}-\ell_{3}$ & $0.8746(25)$ & $0.0186(9)$ & $0.9032(32)$ & $0.0080(6)$ \\
$h_{3}-\ell_{2}$ & $0.8569(38)$ & $0.0166(13)$ & $0.8844(49)$ & $0.0068(7)$ \\
$h_{3}-\ell_{1}$ & $0.8458(56)$ & $0.0150(18)$ & $0.8705(72)$ & $0.0057(9)$ \\
\hline \hline$h_{2}-\ell_{4}$ & $0.8256(16)$ & $0.0221(7)$ & $0.8577(20)$ & $0.0093(4)$ \\
$h_{2}-\ell_{3}$ & $0.7851(23)$ & $0.0175(8)$ & $0.8185(31)$ & $0.0071(5)$ \\
$h_{2}-\ell_{2}$ & $0.7669(34)$ & $0.0157(11)$ & $0.7994(47)$ & $0.0060(6)$ \\
$h_{2}-\ell_{1}$ & $0.7558(48)$ & $0.0144(15)$ & $0.7853(68)$ & $0.0051(8)$ \\
\hline \hline$h_{1}-\ell_{4}$ & $0.7304(13)$ & $0.0199(5)$ & $0.7683(21)$ & $0.0079(3)$ \\
$h_{1}-\ell_{3}$ & $0.6894(19)$ & $0.0161(6)$ & $0.7295(30)$ & $0.0062(3)$ \\
$h_{1}-\ell_{2}$ & $0.6707(27)$ & $0.0145(9)$ & $0.7099(42)$ & $0.0052(4)$ \\
$h_{1}-\ell_{1}$ & $0.6594(37)$ & $0.0136(12)$ & $0.6960(57)$ & $0.0045(5)$ \\
\hline
\end{tabular}

Table 1: Mass spectrum of heavy-light pseudoscalar and vector mesons in lattice units.

We used the standard procedure to extract the pseudoscalar and vector decay constants. This procedure consists in calculating the ratios

$$
\begin{aligned}
\frac{\sum_{\vec{x}}\left\langle\hat{A}_{0}(\vec{x}, t) P(0)\right\rangle}{\sum_{\vec{x}}\langle P(\vec{x}, t) P(0)\rangle} & \simeq \hat{F}_{P} \frac{M_{P}}{\sqrt{\mathcal{Z}}_{P}} \tanh \left(M_{P}\left(\frac{T}{2}-t\right)\right) \\
\sum_{\vec{x}}\left\langle\hat{V}_{i}(\vec{x}, t) \hat{V}_{i}(0)\right\rangle & \simeq M_{V}^{2} \hat{F}_{V}^{2} e^{-M_{V} \frac{T}{2}} \cosh \left(M_{V}\left(\frac{T}{2}-t\right)\right)
\end{aligned}
$$

where we assumed the usual definitions

$$
\begin{aligned}
\left\langle 0\left|\hat{A}_{0}\right| P S(\vec{p}=0)\right\rangle & =i \hat{F}_{P S} M_{P S}, \quad \text { and } \\
\left\langle 0\left|\hat{V}_{i}\right| V(\vec{p}=0 ; \lambda)\right\rangle & =i e_{i}^{(\lambda)} \hat{F}_{V} M_{V} .
\end{aligned}
$$

We denote decay constants and meson masses in lattice units by capital letters, and the hat reminds us that the quantity is improved and renormalized. In practice, one first partially 
improves the bare lattice currents (for clarity, we write the lattice spacing $a$ explicitly):

$$
\begin{aligned}
\left\langle 0\left|A_{0}\right| P S(\vec{p}=0)\right\rangle & \rightarrow\left\langle 0\left|A_{0}\right| P S\right\rangle+c_{A}\left\langle 0\left|a \partial_{0} P\right| P S\right\rangle=i M_{P S}\left(F_{P S}^{(0)}+c_{A} a F_{P S}^{(1)}\right), \\
\left\langle 0\left|V_{i}\right| V(\vec{p}=0 ; \lambda)\right\rangle & \rightarrow\left\langle 0\left|V_{i}\right| V\right\rangle+c_{V}\left\langle 0\left|a \partial_{0} T_{i 0}\right| V\right\rangle=i M_{V} e_{i}^{(\lambda)}\left(F_{V}^{(0)}+c_{V} a F_{V}^{(1)}\right),
\end{aligned}
$$

and then multiplies the currents by suitable overall factors

$$
\begin{array}{ll}
\hat{F}_{P}=Z_{A}\left(1+b_{A} a m\right)\left(F_{P}^{(0)}+c_{A} a F_{P}^{(1)}\right), & {\left[Z_{A}(m)=Z_{A}\left(1+b_{A} a m\right)\right]} \\
\hat{F}_{V}=Z_{V}\left(1+b_{V} a m\right)\left(F_{V}^{(0)}+c_{V} a F_{V}^{(1)}\right), & {\left[Z_{V}(m)=Z_{A}\left(1+b_{V} a m\right)\right] .}
\end{array}
$$

In the calculation of the different correlations above, when the lowest state is well isolated, we may use

$$
\begin{gathered}
\frac{\left\langle\partial_{0} P(t) P(0)\right\rangle}{\langle P(t) P(0)\rangle}=\sinh \left(M_{P S}\right), \\
a F_{P S}^{(1)}=\frac{\sqrt{\mathcal{Z}_{P S}}}{M_{P S}} \sinh \left(M_{P S}\right), \quad \text { and } \quad a F_{V}^{(1)}=\frac{\sqrt{\mathcal{Z}_{T_{i}}}}{M_{V}} \sinh \left(M_{V}\right) .
\end{gathered}
$$

The values of the decay constants are given in Tab. 2.

The improvement coefficients and the renormalization constants are catalogued in Tab. 3, where we also display the one-loop results obtained by using boosted perturbation theory (BPT) at $\beta=6.2[11,20]^{3}$. Recall that the corrective coefficients $b_{J}$ enter with the "average" quark mass defined as $a m=a m_{i j}=\frac{1}{2}\left(a m_{i}+a m_{j}\right)$, where the bare mass is the one derived from the vector Ward identity, namely

$$
a m_{i}=\frac{1}{2}\left(\frac{1}{\kappa_{i}}-\frac{1}{\kappa_{\text {crit }}}\right) .
$$

In the following, we denote by $m_{q}$ and $m_{Q}$ the generic light and heavy quark masses, whereas the quark masses expressed in terms of the corresponding hopping parameters, as in Eq. (16), are denoted by $m_{\ell}$ or $m_{h}$.

Note that, in spite of the non-perturbative determination of $c_{V}$, we used the perturbative value $c_{V}^{B P T}=-0.026$. Firstly, we find the non-perturbative result of Ref. $[8], c_{V}^{N P}=$ $-0.214(74)$, surprising because it is one order of magnitude larger than $c_{V}^{B P T}$. This possibility is not excluded a priori, but it is difficult to accommodate it in the pattern of all other improvement coefficients: when known non-perturbatively, their value is always close to the corresponding (boosted) perturbative one and never differs by one order of magnitude. Secondly, by using $c_{V}^{N P}=-0.214(74)$, the ratio of the vector to the pseudoscalar meson decay constants $f_{H^{*}} / f_{H}$ badly fails in approaching one, as $M_{H}$ increases, contrary to what is predicted by heavy quark symmetry. More details on this scaling law will be given in Sec. 5 . For these reasons, we find it safer to use the $c_{V}^{B P T}$. We believe that the preliminary determination of $c_{V}^{N P}$ in Ref. [8] has some problem and prefer to wait for the final results.

\footnotetext{
${ }^{3}$ This corresponds to the use of $g^{2}=1.256$, in the perturbative formulae.
} 


\begin{tabular}{|c|ccc|crc|}
\hline$\kappa_{1} \kappa_{2}$ & $F_{P S}^{(0)}$ & $-c_{A} a F_{P S}^{(1)} / F_{P S}^{(0)}$ & $F_{P S}$ & $F_{V}^{(0)}$ & $-c_{V} a F_{V}^{(1)} / F_{V}^{(0)}$ & $F_{V}$ \\
\hline \hline$h_{4}-\ell_{4}$ & $0.0957(16)$ & $0.0730(6)$ & $0.0887(15)$ & $0.1043(21)$ & $0.0276(2)$ & $0.1014(21)$ \\
$h_{4}-\ell_{3}$ & $0.0869(23)$ & $0.0702(6)$ & $0.0917(29)$ & $0.0942(30)$ & $0.0260(4)$ & $0.0740(23)$ \\
$h_{4}-\ell_{2}$ & $0.0825(35)$ & $0.0694(8)$ & $0.0859(42)$ & $0.0881(44)$ & $0.0251(6)$ & $0.0698(34)$ \\
$h_{4}-\ell_{1}$ & $0.0793(48)$ & $0.0687(10)$ & $0.0803(58)$ & $0.0823(60)$ & $0.0245(9)$ & $0.0658(48)$ \\
\hline$h_{3} \ell_{4}$ & $0.0982(15)$ & $0.0664(6)$ & $0.0917(14)$ & $0.1089(21)$ & $0.0235(2)$ & $0.1063(20)$ \\
$h_{3}-\ell_{3}$ & $0.0896(21)$ & $0.0639(6)$ & $0.0839(20)$ & $0.0990(30)$ & $0.0221(3)$ & $0.0969(29)$ \\
$h_{3}-\ell_{2}$ & $0.0853(30)$ & $0.0630(8)$ & $0.0799(28)$ & $0.0928(42)$ & $0.0214(5)$ & $0.0908(41)$ \\
$h_{3}-\ell_{1}$ & $0.0822(42)$ & $0.0625(10)$ & $0.0771(39)$ & $0.0870(58)$ & $0.0208(7)$ & $0.0852(57)$ \\
\hline$h_{2}-\ell_{4}$ & $0.0999(14)$ & $0.0615(6)$ & $0.0938(14)$ & $0.1126(21)$ & $0.0206(2)$ & $0.1103(20)$ \\
$h_{2}-\ell_{3}$ & $0.0916(19)$ & $0.0591(6)$ & $0.0862(18)$ & $0.1032(29)$ & $0.0193(3)$ & $0.1012(29)$ \\
$h_{2}-\ell_{2}$ & $0.0875(27)$ & $0.0583(8)$ & $0.0824(25)$ & $0.0969(41)$ & $0.0186(4)$ & $0.0951(40)$ \\
$h_{2}-\ell_{1}$ & $0.0846(37)$ & $0.0578(9)$ & $0.0797(35)$ & $0.0910(57)$ & $0.0181(6)$ & $0.0894(56)$ \\
\hline$h_{1}-\ell_{4}$ & $0.1009(15)$ & $0.0564(5)$ & $0.0953(15)$ & $0.1154(20)$ & $0.0178(1)$ & $0.1134(19)$ \\
$h_{1}-\ell_{3}$ & $0.0933(18)$ & $0.0544(6)$ & $0.0883(18)$ & $0.1078(29)$ & $0.0165(2)$ & $0.1060(29)$ \\
$h_{1}-\ell_{2}$ & $0.0895(24)$ & $0.0536(8)$ & $0.0847(23)$ & $0.1015(40)$ & $0.0159(3)$ & $0.0999(39)$ \\
$h_{1}-\ell_{1}$ & $0.0870(32)$ & $0.0531(9)$ & $0.0823(30)$ & $0.0957(55)$ & $0.0155(5)$ & $0.0942(54)$ \\
\hline
\end{tabular}

Table 2: Heavy-light decay constants in lattice units.

\section{$3 \quad D$-meson spectrum and decay constants}

In this section, we discuss the $D$-meson spectrum and decay constants. Preliminary results of this study were given in Ref. [22]

In Tab. 4 , we tabulate the results for the heavy-light meson masses, $M_{H}\left(m_{h}, m_{\ell}\right)$, obtained from a linear extrapolation (interpolation) in the light quark mass (to reach $q=u, d$ and $s$ ). This was achieved by using the method of physical lattice planes. In Ref. [17], we extracted the $M_{\pi}$, and the hypothetical pseudoscalar $M_{\eta_{s s}}$, which (when squared) are proportional to $m_{q}$ and $m_{s}$, respectively. For the generic physical quantity in the heavy-light meson sector $\mathfrak{F}_{H}\left(m_{h}, m_{\ell}\right)$, we use the following form of fit

$$
\mathfrak{F}_{H}\left(m_{h}, m_{\ell_{i}}\right)=\alpha_{h}+\beta_{h} M_{P S}^{2}\left(m_{\ell_{i}}, m_{\ell_{i}}\right)+\gamma_{h}\left(M_{P S}^{2}\left(m_{\ell_{i}}, m_{\ell_{i}}\right)\right)^{2},
$$

\footnotetext{
${ }^{4}$ See [23] for preliminary results from the UKQCD collaboration and the APETOV group.
} 


\begin{tabular}{|c|c|c|}
\hline \multicolumn{3}{|c|}{$\begin{array}{l}\text { Renormalization constants } \\
\text { (in the chiral limit) }\end{array}$} \\
\hline $\begin{array}{c}\text { Quantity } \\
\text { BPT } \\
\text { Non - perturbative } \\
\end{array}$ & $\begin{array}{c}Z_{V} \\
0.846 \\
\mathbf{0 . 7 9 3}\end{array}$ & $\begin{array}{c}Z_{A} \\
0.862 \\
\mathbf{0 . 8 0 9}\end{array}$ \\
\hline \multicolumn{3}{|c|}{$\begin{array}{l}\text { Coefficients for the Improvement } \\
\text { of the Bare Operators }\end{array}$} \\
\hline $\begin{array}{c}\text { Quantity } \\
\text { BPT } \\
\text { Non-perturbative }\end{array}$ & $\begin{array}{c}c_{V} \\
\mathbf{- 0 . 0 2 6} \\
-0.214(74) \\
\end{array}$ & $\begin{array}{c}c_{A} \\
-0.012 \\
\mathbf{- 0 . 0 3 7}\end{array}$ \\
\hline \multicolumn{3}{|c|}{$\begin{array}{l}\text { Coefficients for the Renormalization Constants } \\
\text { Improvement (due to the Explicit Mass Term) }\end{array}$} \\
\hline $\begin{array}{c}\text { Quantity } \\
\text { BPT } \\
\text { Non-perturbative }\end{array}$ & $\begin{array}{c}b_{V} \\
1.242 \\
\mathbf{1 . 4 0 4}\end{array}$ & $\begin{array}{c}b_{A} \\
\mathbf{1 . 2 4 0} \\
\text { NOT CALC. }\end{array}$ \\
\hline
\end{tabular}

Table 3: Improvement coefficients. In boosted perturbation theory $g^{2}=1.256$. For the perturbative $Z_{J}$ 's, we used $c_{S W}=1.614$. The values which have been used in our numerical calculations are marked in bold.

where the heavy quark mass (i.e. $\left.\kappa_{h}\right)$ is kept fixed. The coefficients of such a fit, $\alpha_{h}, \beta_{h}$, $\gamma_{h}$, are then used to obtain $\mathfrak{F}_{H}\left(m_{h}, m_{q}\right)$ and $\mathfrak{F}_{H}\left(m_{h}, m_{s}\right)$, by inserting on the r.h.s. of (17), $M_{\pi}^{2}$ and $M_{\eta_{s s}}^{2}$, respectively. In practice, it turns out that the linear $\left(\gamma_{h}=0\right)$ and quadratic $\left(\gamma_{h} \neq 0\right)$ fits give essentially the same results for any physical quantity considered in this study . In Fig. 2, we show this effect for the pseudoscalar decay constant. Therefore, in all results that we present in what follows, whenever a quantity with light quark flavor $q$ and/or $s$ is mentioned, it means that the linear fit in Eq. (17) is performed, i.e. $\gamma_{h}=0$.

Having fixed the light quark mass, we now want to interpolate in the heavy quark mass. In the framework of the HQET, the functional dependence of $M_{H}$ on $m_{Q}$ is:

$$
M_{H}=m_{Q}\left\{1+\frac{\bar{\Lambda}}{m_{Q}}+\frac{1}{2 m_{Q}^{2}}\left(\lambda_{1}+k \lambda_{2}\right)+\mathcal{O}\left(\frac{1}{m_{Q}^{3}}\right)\right\}
$$

where $\bar{\Lambda}$ is the so-called binding energy, $\lambda_{1,2}$ are the first (flavor-spin) symmetry breaking corrections (describing the kinetic and chromomagnetic energy), and $k=3(-1)$, for $J^{P}=0^{-}\left(1^{-}\right)$. The improvement of the quark mass brings in the quadratic terms in $m_{h}$, i.e. $m_{h} \rightarrow m_{h}\left(1+b_{m} m_{h}\right)$, and distort all the coefficients in the expansion (18). The term of order $m_{h}^{2}$ originates only from the lattice artifacts, and thus is always proportional to $b_{m}$. The interplay between power corrections in $1 / m_{Q}$ and discretization effects, however,

\footnotetext{
${ }^{5}$ As expected, the results obtained from a quadratic fit inflates the errors in extrapolated results.
} 


\begin{tabular}{|c|c|c|c|c|}
\hline "Flavor" content & $\mathrm{M}_{\mathrm{PS}}$ & $\hat{F}_{\mathrm{PS}}$ & $\mathrm{M}_{\mathrm{V}}$ & $\hat{F}_{\mathrm{V}}$ \\
\hline \hline$h_{4}-s$ & $0.9721(61)$ & $0.0870(36)$ & $0.9947(62)$ & $0.0992(49)$ \\
$h_{4}-q$ & $0.9414(58)$ & $0.0783(47)$ & $0.9635(65)$ & $0.0875(61)$ \\
\hline$h_{3}-s$ & $0.8595(56)$ & $0.0856(30)$ & $0.8871(61)$ & $0.0986(45)$ \\
$h_{3}-q$ & $0.8281(50)$ & $0.0774(39)$ & $0.8555(62)$ & $0.0877(56)$ \\
\hline$h_{2}-s$ & $0.7697(53)$ & $0.0845(26)$ & $0.8022(61)$ & $0.0985(43)$ \\
$h_{2}-q$ & $0.7375(43)$ & $0.0768(33)$ & $0.7702(59)$ & $0.0881(53)$ \\
\hline$h_{1}-s$ & $0.6736(49)$ & $0.0831(22)$ & $0.7126(62)$ & $0.0985(40)$ \\
$h_{1}-q$ & $0.6409(35)$ & $0.0761(29)$ & $0.6804(54)$ & $0.0896(51)$ \\
\hline
\end{tabular}

Table 4: Mass spectrum and decay constants of heavy-light pseudoscalar and vector mesons. $h_{i}-s$ and $h_{i}-q$ denote mesons composed by a heavy quark with mass $m_{h_{i}}$ and a strange or a light $(u, d)$ quark, respectively. All the results are expressed in lattice units.

modifies the "effective" value of $b_{m}$, i.e. the coefficient of the quadratic term in $m_{h}$. To investigate this point, we study the behaviour of $M_{H}$ in $m_{h}$, at fixed light quark mass, $m_{q}$. In the $D$ case, we use the following expression:

$$
M_{H}\left(m_{h_{i}}, m_{q}\right)-a m_{D}=\mathcal{A}\left(m_{h_{i}}-m_{\text {charm }}\right)\left[1+\mathcal{B}\left(m_{h_{i}}+m_{\text {charm }}\right)\right]
$$

where $a m_{D}$ is the experimental meson mass in lattice units, $a m_{D}=0.68(4)$ (similarly we fit $M_{H^{*}}\left(m_{h_{i}}, m_{q}\right)-a m_{D^{*}}$, etc.). From the fit of our data to Eq. (19), it turns out that the resulting value for $\kappa_{\text {charm }}$ is stable for $\mathcal{B} \in[-0.4,-0.2]$. The minimum $\chi^{2}$ is reached for $\mathcal{B}=-0.32$. We have also performed the linear fit (corresponding to $\mathcal{B}=0$ ), and the fit with $\mathcal{B}=b_{m}^{B P T}=-0.652$ [1]. The different values that we obtain for $\kappa_{\text {charm }}$ with different fits (linear, quadratic or using $b_{m}^{B P T}$ ) differ by about one per mille. We quote

$$
\kappa_{\text {charm }}=0.1231(14) \text {. }
$$

It can be argued that a fit of the spin-average mass $\bar{M}_{H}=\left(3 M_{V}+M_{P S}\right) / 4$, to extract $\kappa_{\text {charm }}$ is more suitable, because spin forces of $\mathcal{O}\left(1 / m_{Q}\right)$ are canceled in this combination (see (18)). For $\mathcal{B}=-0.32$, corresponding also in this case to the minimum $\chi^{2}$, we obtain $\kappa_{\text {charm }}=0.1232(14)$. Since the differences for the $D$-meson masses and decay constants as obtained by using the two values of $\kappa_{\text {charm }}$ is very small, in the following, whenever we refer 


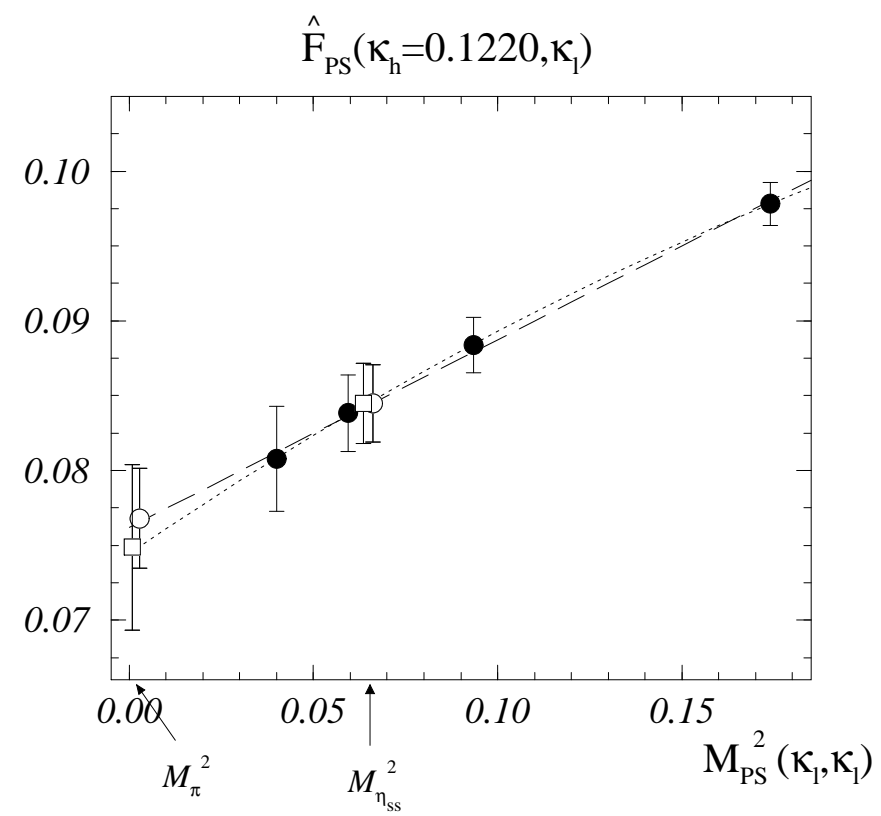

Figure 2: Fits of $\hat{F}_{P S}$ in the light quark mass, at fixed $m_{h}$. The filled circles denote data directly measured. The dashed curve and empty circles refer to the linear fit and extrapolated points. The dotted curve and empty squares to the quadratic fit and extrapolated points. The heavy quark mass corresponds to $\kappa_{h_{2}}$.

to $\kappa_{\text {charm }}$, the value $(\sqrt[20]{ })$ is understood. Using $M_{D}$ as a physical input (to fix $\kappa_{\text {charm }}$ ), we can make several predictions for other meson masses

$$
\begin{array}{rll}
M_{D} \equiv \text { input } & ; & M_{D^{*}}=0.725(42), \quad \text { and } \\
M_{D_{s}}=0.733(46) \quad ; & M_{D_{s}^{*}}=0.768(45) .
\end{array}
$$

which in physical units give

$$
m_{D^{*}}=1.992(24) \mathrm{GeV}, \quad m_{D_{s}}=2.013(18) \mathrm{GeV}, \quad m_{D_{s}^{*}}=2.110(21) \mathrm{GeV},
$$

to be compared to the experimental numbers [24]

$$
m_{D^{*}}^{(\text {exp. })}=2.008 \mathrm{GeV} ; \quad m_{D_{s}}^{(e x p .)}=1.968 \mathrm{GeV} ; \quad m_{D_{s}^{*}}^{(e x p .)}=2.112(27) \mathrm{GeV} .
$$

We obviously fail to obtain the experimentally measured mass-difference. We get

$$
m_{D_{s}^{*}}-m_{D_{s}}=(97 \pm 12) \mathrm{MeV} .
$$

which is to be compared to $\left(m_{D_{s}^{*}}-m_{D_{s}}\right)^{(\exp .)}=143.8(4) \mathrm{MeV}$. 
An alternative procedure is to consider the ratio $C_{V V} / C_{P P}$, from which the vectorpseudoscalar mass difference can be directly extracted. By using this method we get

$$
\begin{gathered}
M_{D^{*}}-M_{D}=0.0354(39) \quad \rightarrow \quad m_{D^{*}}-m_{D}=97(15) \mathrm{MeV} \\
M_{D_{s}^{*}}-M_{D_{s}}=0.0354(30) \quad \rightarrow \quad m_{D_{s}^{*}}-m_{D_{s}}=97(13) \mathrm{MeV} ;
\end{gathered}
$$

which confirms that the ('spin') mass difference is systematically smaller than the experimental one, regardless of the procedure we use. Since we found a reasonable agreement for the hyperfine splitting in the light-quark sector [17], the discrepancy in the heavy-quark case is probably a signal of large residual $\mathcal{O}\left(a^{2}\right)$ errors. We believe that the discrepancy cannot be entirely attributed to the use of the quenched approximation 0 .

Our results for the hyperfine splitting are shown in Fig. 3. From that figure, we note the qualitative agreement between the dependence of the splitting on the light-quark mass measured on the lattice and its experimental counterpart. Moreover, the dependence of the hyperfine splitting on the meson mass is not dramatically larger than the experimental one, represented by a gray line in the figure. This is to be contrasted to the case of the unimproved Wilson action, where the lattice slope is by far larger than in the present case [21], showing a clear effect of improvement, although insufficient to describe the experimental numbers. In Tab. 5, we list the results extrapolated in the light quark mass, at fixed $m_{h}$.

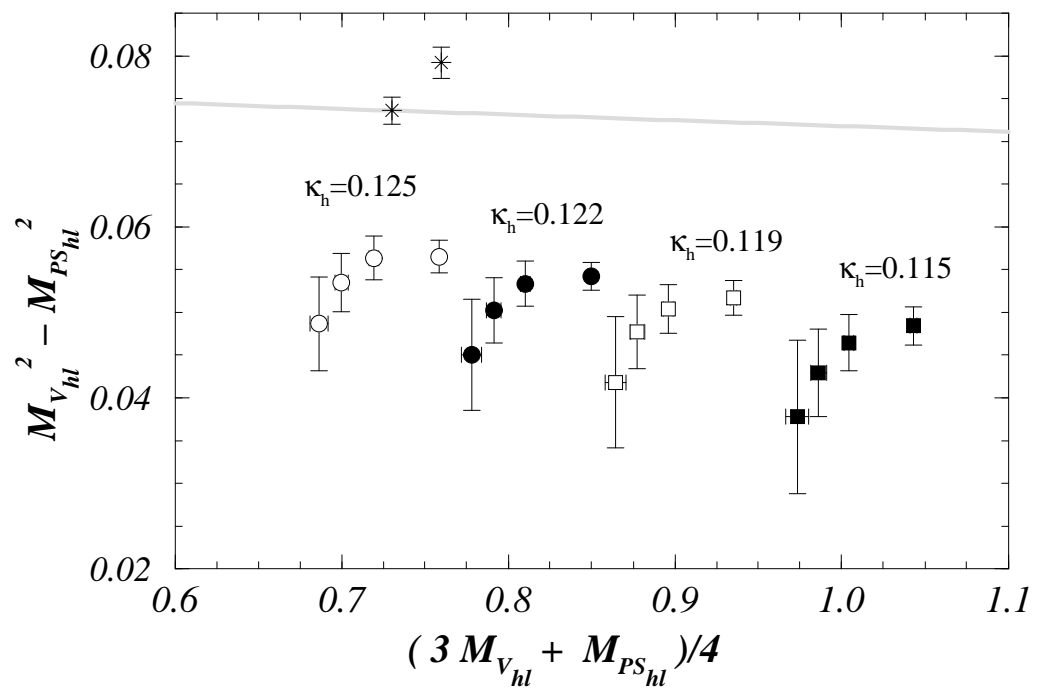

Figure 3: Hyperfine splitting for heavy-light mesons in lattice units. The gray line shows the (approximate) experimental slope. The stars mark the physical $m_{D^{*}}^{2}-m_{D}^{2}$ and $m_{D_{s}^{*}}^{2}-m_{D_{s}}^{2}$ splittings.

\footnotetext{
${ }^{6}$ Quenching is always a joker-argument when we are unable to solve or explain a problem in lattice calculations.
} 


\begin{tabular}{|c|c|c|}
\hline heavy & $q$ & $s$ \\
\hline$h_{1}$ & $0.393(53)$ & $0.406(50)$ \\
$h_{2}$ & $0.367(57)$ & $0.383(52)$ \\
$h_{3}$ & $0.342(61)$ & $0.361(54)$ \\
$h_{4}$ & $0.310(68)$ & $0.330(57)$ \\
\hline
\end{tabular}

Table 5: Hyperfine splitting, $M_{H^{*}}^{2}-M_{H}^{2}$ in $\mathrm{GeV}^{2}$.

We now discuss the physical results for the $D$-meson decay constants. We first extrapolate the results, in the light quark mass, at fixed $m_{h}$, by using a formula similar to Eq. (17), i.e.

$$
\hat{F}_{H}\left(m_{h}, m_{\ell_{i}}\right)=\alpha_{h}^{F}+\beta_{h}^{F} M_{P S}^{2}\left(m_{\ell_{i}}, m_{\ell_{i}}\right) .
$$

The results of this extrapolation, $m_{\ell_{i}} \rightarrow m_{q}$ and $m_{\ell_{i}} \rightarrow m_{s}$, are given in Tab. 1 .

To handle the problem of extrapolation in the heavy-quark mass, at fixed light-quark mass, we rely on the heavy quark symmetry. The relevant scaling law is

$$
f_{H}=\frac{\Phi\left(m_{H}\right)}{\sqrt{m_{H}}}\left(1+\frac{\Phi^{\prime}\left(m_{H}\right)}{\Phi\left(m_{H}\right) m_{H}}+\ldots\right)
$$

where $\Phi\left(m_{H}\right), \Phi^{\prime}\left(m_{H}\right)$ depend logarithmically on the mass, e.g. $\Phi\left(m_{H}\right) \sim \alpha_{s}^{-2 / b_{0}}\left(m_{H}\right)(1+$ $\left.\mathcal{O}\left(\alpha_{s}\right)\right)$ ๆ. In the interval of masses considered in this study, the logarithmic corrections are negligible. For this reason, in our fits, we used

$$
\hat{F}_{H} \sqrt{M_{H}}=\Phi_{0}+\frac{\Phi_{1}}{M_{H}}+\frac{\Phi_{2}}{M_{H}^{2}},
$$

where $\Phi_{0}, \Phi_{1}$ and $\Phi_{2}$ are constants. At the physical point $M_{H}=a m_{D}$ (corresponding to $\kappa_{h}=\kappa_{\text {charm }}$ ), we read off the value $\hat{F}_{D}$ in lattice units. To express it in physical units, one simply multiplies by $a^{-1}$. The same procedure can be used for the vector-meson decay constants.

Another possibility, is to consider the ratios $\hat{R}_{H}\left(m_{h}, m_{\ell}\right)=\hat{F}_{H}\left(m_{h}, m_{\ell}\right) / \hat{F}_{P S}\left(m_{q}, m_{\ell}\right)$ and $\hat{R}_{H^{*}}=\hat{F}_{H^{*}}\left(m_{h}, m_{\ell}\right) / \hat{F}_{V}\left(m_{q}, m_{\ell}\right)$, and to extrapolate $\hat{R}_{H}\left(m_{h}, m_{\ell}\right)$ in $m_{\ell}$ and $m_{h}$ by using eqs. (25) and (27), with the obvious replacement $\hat{F}_{H} \rightarrow \hat{R}_{H}\left(\hat{F}_{\pi, \rho}=\hat{F}_{P S, V}\left(m_{q}, m_{q}\right)\right.$, $\left.\hat{F}_{K, K^{*}}=\hat{F}_{P S, V}\left(m_{q}, m_{s}\right)\right)$. The physical values of the decay constants are then obtained by using

$$
f_{D}=\hat{R}_{H}\left(m_{\text {charm }}, m_{q}\right) \times f_{\pi}^{(\text {exp. })}, \quad f_{D_{s}}=\hat{R}_{H_{s}}\left(m_{\text {charm }}, m_{s}\right) \times f_{K}^{(\text {exp. })},
$$

\footnotetext{
${ }^{7}$ We prefer to give the scaling law in terms of the hadron mass $m_{H}$ rather than the heavy quark mass.
} 
and similarly for the vector mesons

$$
f_{D^{*}}=\hat{R}_{H^{*}}\left(m_{\text {charm }}, m_{q}\right) \times f_{\rho}^{(\text {exp. })}, \quad f_{D_{s}^{*}}=\hat{R}_{H_{s}^{*}}\left(m_{\text {charm }}, m_{s}\right) \times f_{K^{*}}^{(\text {exp. })} .
$$

The experimental values of the decay constants that we use are the following ones [24]: $f_{\pi}^{(\text {exp. })}=131 \mathrm{MeV}, f_{K}^{(\text {exp. })}=160 \mathrm{MeV}, f_{\rho}^{(\text {exp. })}=208(2) \mathrm{MeV}, f_{K^{*}}^{(\text {exp. })}=214(7) \mathrm{MeV}$.

The results are given in Tab. 6. We also give the decay constants obtained by including the KLM factor which we discuss in Sec. 1 . The differences can be used for an estimate of the residual $\mathcal{O}\left(a^{2}\right)$, discretization errors in the determination of the matrix elements. In Tab. 7 , we list the corresponding results for vector mesons.

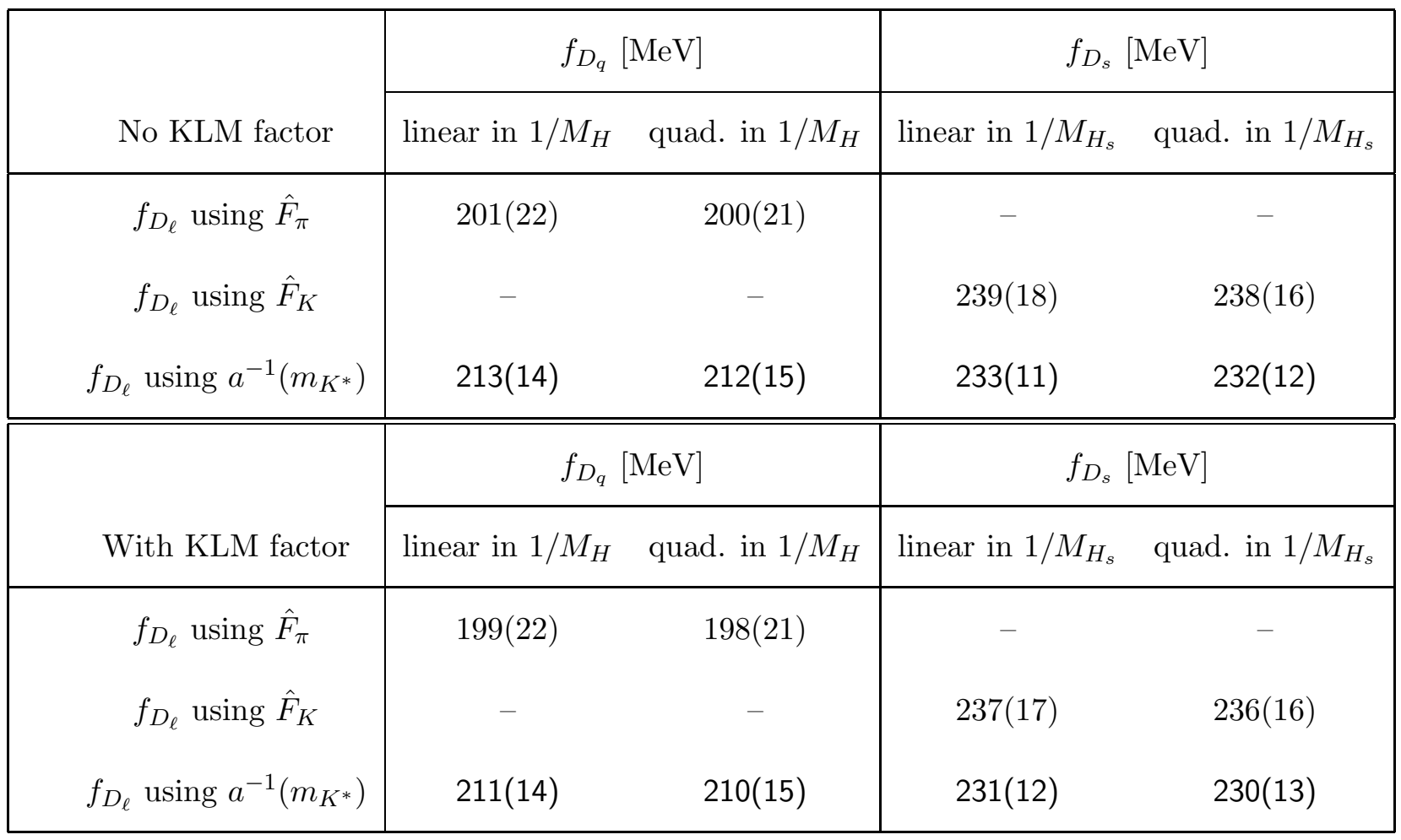

Table 6: Pseudoscalar decay constants for D-mesons using the scaling law in Eq. (27). Results including the KLM factor discussed in the text, are given in the lower part of the table.

Whether we use a linear or a quadratic fit to interpolate to $\kappa_{\text {charm }}$, our results in the $D$-sector remain practically unchanged. In order to illustrate the stability of the results for $D$ mesons, we also show in Fig. 4 , the results of the linear and quadratic fits in $1 / M_{H}$.

The observed stability makes these results quite remarkable: we use the non-perturbatively improved action; the operators and the renormalization constants are also improved; the results obtained by using the heavy quark scaling laws are unchanged, regardless of whether we take quadratic $\left(1 / M_{H}^{2}\right)$ corrections into account or not; the results are practically insensitive to the presence of KLM factors; there is no important dependence on the quantity chosen 

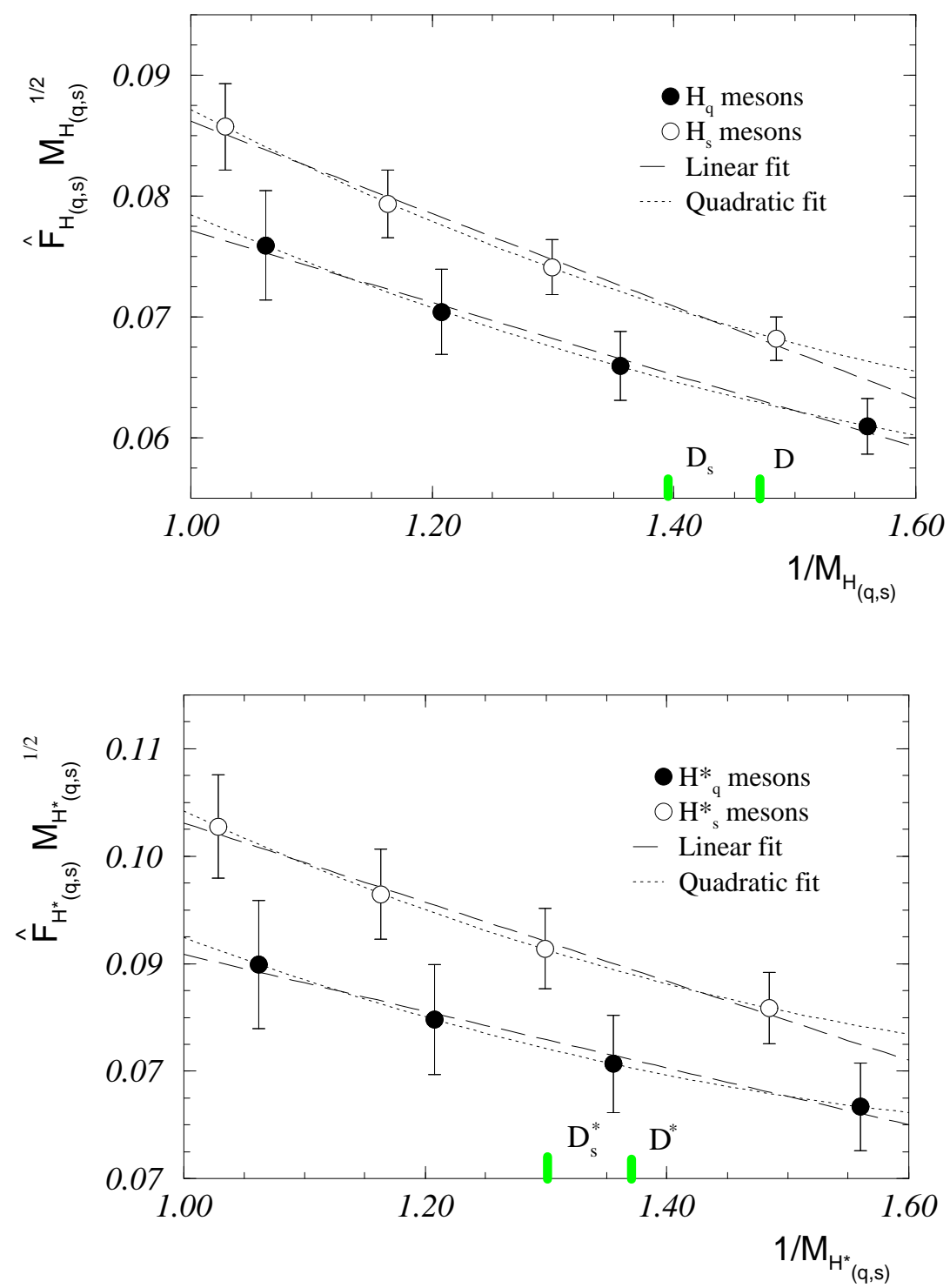

Figure 4: Results of the linear and quadratic fits for pseudoscalar and vector mesons, in lattice units. The gray dashed lines correspond to $f_{D}\left(f_{D_{s}}\right)$, and $f_{D^{*}}\left(f_{D_{s}^{*}}\right)$ respectively. 


\begin{tabular}{|c|c|c|c|c|}
\hline \multirow[b]{2}{*}{ No KLM factor } & \multicolumn{2}{|c|}{$f_{D_{q}^{*}}[\mathrm{MeV}]$} & \multicolumn{2}{|c|}{$f_{D_{s}^{*}}[\mathrm{MeV}]$} \\
\hline & linear in $1 / M_{l}$ & quad. in $1 / M_{H}$ & linear in $1 / M_{H_{s}}$ & quad. in $1 / M_{H_{s}}$ \\
\hline$f_{D_{\ell}^{*}}$ using $\hat{F}_{\rho}$ & $246(30)$ & $244(32)$ & - & - \\
\hline$f_{D_{\ell}^{*}}$ using $\hat{F}_{K^{*}}$ & - & - & $255(17)$ & $253(18)$ \\
\hline \multirow[t]{2}{*}{$f_{D_{\ell}^{*}}$ using $a^{-1}\left(m_{K^{*}}\right)$} & $248(19)$ & $246(21)$ & $275(15)$ & $273(16)$ \\
\hline & \multicolumn{2}{|c|}{$f_{D_{q}^{*}}[\mathrm{MeV}]$} & \multicolumn{2}{|c|}{$f_{D_{s}^{*}}[\mathrm{MeV}]$} \\
\hline With KLM factor & linear in $1 / M_{1}$ & quad. in $1 / M_{H}$ & linear in $1 / M_{H_{s}}$ & quad. in $1 / M_{H_{s}}$ \\
\hline$f_{D_{\ell}^{*}}$ using $\hat{F}_{\rho}$ & $243(31)$ & $241(33)$ & - & - \\
\hline$f_{D_{\ell}^{*}}$ using $\hat{F}_{K^{*}}$ & - & - & $252(18)$ & $251(19)$ \\
\hline$f_{D_{\ell}^{*}} \operatorname{using} a^{-1}\left(m_{K^{*}}\right)$ & $245(20)$ & $243(21)$ & $272(16)$ & $270(16)$ \\
\hline
\end{tabular}

Table 7: Vector decay constants for charmed-mesons obtained by using the scaling law in Eq. (27). Results, including the KLM factor discussed in the text, are also given.

to fix the physical normalization. The errors that we quoted in (11), are obtained in the following way: a central value is fixed by the result obtained from the linear fit in $1 / M_{H}$, with the scale fixed by $m_{K^{*}}$, and the KLM factor included; we quote the statistical error as estimated using the jackknife procedure; all the residual differences are lumped into the systematic uncertainty (the difference between the central values of the results obtained by using different quantities for the scale fixing, and the difference with the central value of the result obtained from the quadratic fit in $1 / M_{H}$ ). It is also worth noticing the remarkable stability of the ratio $f_{D_{s}} / f_{D}$ (see Eq. (1D)), although it may be questioned whether we are really able to predict the $\mathrm{SU}(3)$ breaking properly in the quenched approximation. More discussion on this point will be given in Sec. $\mathrm{F}$.

\section{$4 \quad B$ mesons}

In this section, we present the results of the extrapolation of the decay constants to the $B$ mesons, and discuss the discretization errors in the extrapolation.

When extrapolating the raw data obtained for $m_{h} \sim m_{\text {charm }}$ to the $B$-sector, two important effects may arise. On the one hand, the inclusion of the quadratic term $\Phi_{2} / M_{H}^{2}$ in 
Eq. (27) may change appreciably the results of the linear fit, on the other the $\mathcal{O}(a)$ corrections $\left(\mathcal{O}(a)\right.$ terms proportional to $c_{V, A}$ and $b_{V, A}$ in (13)) become much larger. This is to be contrasted to the case of $D$-mesons, where the inclusion of the quadratic corrections leaves the results essentially unchanged, cf. Tabs. 6 and 7 .

The effect of $c_{A}, c_{V}, b_{V}$ and $b_{A}$ is sizable for the scaling behaviour of $f_{B, B^{*}}$. Note also that if we used $c_{V}^{N P}$, this effect would be huge for the vector decay constant. For instance, in the range of quark masses considered in our simulation, the renormalization constants $Z_{V, A}(m)$, defined in Eq. (13), increase by $20 \div 50 \%$, relatively to their values in the chiral limit. Since $Z_{V}(m)$ and $Z_{A}(m)$ are multiplicative factors, their effect is very important for the extrapolation to the $B$-sector. This is illustrated in Fig. 5: when $Z_{A}(m)$ is included, we note that the quadratic fit is more desired, although the linear one is compatible with the data. The embarrassing point is that the values of $f_{B}$ and $f_{B^{*}}$, as obtained from the linear and quadratic fit, are hardly compatible, see Tabs. 8 and 9 . This is particularly pronounced for $B_{s}^{(*)}$ mesons.

\begin{tabular}{|c|c|c|c|c|}
\hline \multirow[b]{2}{*}{ No KLM factor } & \multicolumn{2}{|c|}{$f_{B_{q}}[\mathrm{MeV}]$} & \multicolumn{2}{|c|}{$f_{B_{s}}[\mathrm{MeV}]$} \\
\hline & \multicolumn{2}{|c|}{ linear in $1 / M_{H} \quad$ quad. in $1 / M_{H}$} & \multicolumn{2}{|c|}{ linear in $1 / M_{H_{s}}$ quad. in $1 / M_{H_{s}}$} \\
\hline$f_{B_{\ell}}$ using $\hat{F}_{\pi}$ & $176(19)$ & $208(27)$ & - & - \\
\hline$f_{B_{\ell}}$ using $\hat{F}_{K}$ & - & - & $217(14)$ & $255(20)$ \\
\hline \multirow[t]{2}{*}{$f_{B_{\ell}}$ using $a^{-1}\left(m_{K^{*}}\right)$} & $187(19)$ & $220(25)$ & $212(16)$ & $249(20)$ \\
\hline & \multicolumn{2}{|c|}{$f_{B_{q}}[\mathrm{MeV}]$} & \multicolumn{2}{|c|}{$f_{B_{s}}[\mathrm{MeV}]$} \\
\hline With KLM factor & linear in $1 / M$ & quad. in $1 / M_{H}$ & linear in $1 / M_{H_{s}}$ & d. in $1 / M_{H_{s}}$ \\
\hline$f_{B_{\ell}}$ using $\hat{F}_{\pi}$ & $170(18)$ & $193(25)$ & - & - \\
\hline$f_{B_{\ell}}$ using $\hat{F}_{K}$ & - & - & $209(13)$ & $238(19)$ \\
\hline$f_{B_{\ell}}$ using $a^{-1}\left(m_{K^{*}}\right)$ & $179(18)$ & $205(24)$ & 204(16) & 232(19) \\
\hline
\end{tabular}

Table 8: Pseudoscalar decay constants for B-mesons using the scaling law in Eq. (27). Results with the KLM factor included are listed in lower part of the table.

The curvature in the fit to $f_{B}$ could partially be induced by $\mathcal{O}\left(a^{2}\right)$ terms, still present in the calculation of the matrix elements. A possible way to account for some of these effects is through the so-called KLM factor [25]. In our case, this means that, besides the factor 

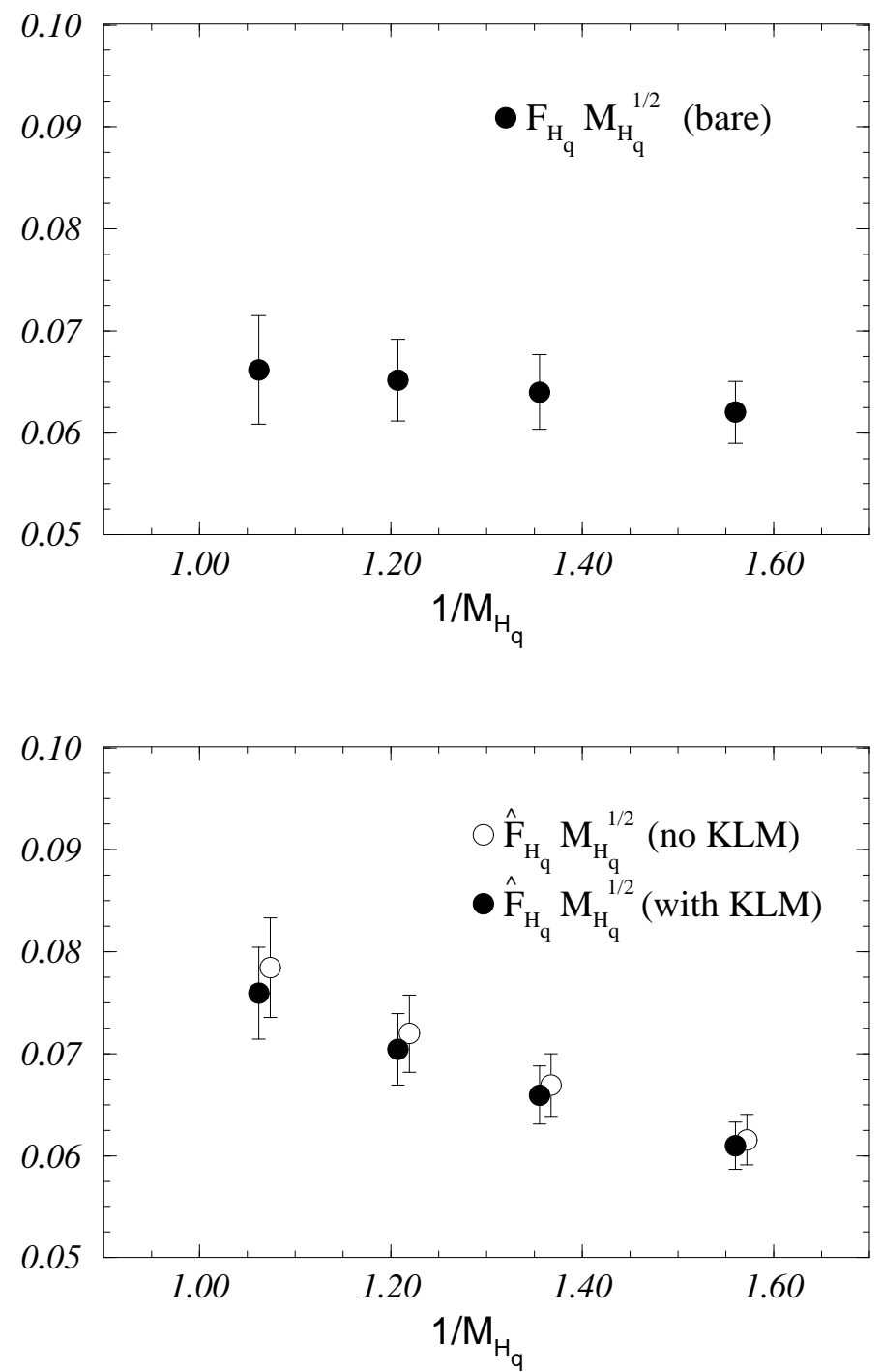

Figure 5: Heavy-light pseudoscalar decay constant as a function of $1 / M_{H}$. The two figures illustrate the influence of the renormalization constant $Z_{A}(m)$ on the $1 / M_{H}$ dependence of the decay constant. 


\begin{tabular}{|c|c|c|c|c|}
\hline \multirow[b]{2}{*}{ No KLM factor } & \multicolumn{2}{|c|}{$f_{B_{q}^{*}}[\mathrm{MeV}]$} & \multicolumn{2}{|c|}{$f_{B_{s}^{*}}[\mathrm{MeV}]$} \\
\hline & linear in $1 / M_{l}$ & quad. in $1 / M_{H}$ & linear in $1 / M_{H_{s}}$ & quad. in $1 / M_{H_{s}}$ \\
\hline$f_{B_{\ell}^{*}}$ using $\hat{F}_{\rho}$ & $204(34)$ & $239(39)$ & - & - \\
\hline$f_{B_{\ell}^{*}}$ using $\hat{F}_{K^{*}}$ & - & - & $222(22)$ & $260(25)$ \\
\hline \multirow[t]{2}{*}{$f_{B_{\ell}^{*}}$ using $a^{-1}\left(m_{K^{*}}\right)$} & $205(25)$ & $241(32)$ & $239(21)$ & $280(24)$ \\
\hline & \multicolumn{2}{|c|}{$f_{B_{q}^{*}}[\mathrm{MeV}]$} & \multicolumn{2}{|c|}{$f_{B_{s}^{*}}[\mathrm{MeV}]$} \\
\hline With KLM factor & linear in $1 / M_{l}$ & quad. in $1 / M_{H}$ & linear in $1 / M_{H_{s}}$ & quad. in $1 / M_{H_{s}}$ \\
\hline$f_{B_{\ell}^{*}}$ using $\hat{F}_{\rho}$ & $194(32)$ & $225(37)$ & - & - \\
\hline$f_{B_{\ell}^{*}}$ using $\hat{F}_{K^{*}}$ & - & - & $213(22)$ & $241(24)$ \\
\hline$f_{B_{\ell}^{*}}$ using $a^{-1}\left(m_{K^{*}}\right)$ & $196(24)$ & $227(30)$ & $229(20)$ & $260(23)$ \\
\hline
\end{tabular}

Table 9: Vector decay constants for B-mesons using the scaling law in Eq. (27). Results including the KLM factor discussed in the text, are also given.

$\left(1+b_{J} m a\right)$ already included in the definition of the renormalized currents (13), we may try to include the effects of higher order terms in $m a$, by using the following relation

$$
\begin{aligned}
Z_{J}\left(m_{h}, m_{\ell}\right) & =Z_{J}(0)\left[\frac{\sqrt{1+a m_{h}} \sqrt{1+a m_{\ell}}}{1+a m}\right]\left(1+b_{J} a m\right) \\
& \simeq Z_{J}(0)\left(1+b_{J} a m\right)+\mathcal{O}\left(a^{2}\right),
\end{aligned}
$$

where $a m=\left(a m_{h}+a m_{\ell}\right) / 2$, and $a m_{i}$ is the usual expression for the bare quark mass (16). Equation (30) is a consequence of the redefinition of a quark field, $q \rightarrow \sqrt{1+a m} q$ (in the KLM way), which comes from the comparison of the free lattice quark propagator to its continuum counterpart. The results which include the KLM correction are given in the lower part of Tabs. 6, 7, 8 and 9. In the case of $D$-mesons, the effect of KLM is indeed negligible. In the case of $B$-mesons, we observe a slight change in the central values, e.g. $f_{B}=187 \mathrm{MeV} \rightarrow 179 \mathrm{MeV}$. However, the distance between the values obtained with linear and with quadratic fits remains essentially unchanged. In the absence of a larger range of masses, we are unable to reduce the difference between results obtained with the linear and quadratic fits. As it has been done for $D$-mesons, we quote the results of the linear fits as our central values, and include in the systematic error the differences between our central values 
and $i$ ) the results from the quadratic fit; $i i$ ) the results without the KLM factor incorporated; iii) the results obtained by using other quantities $\left(f_{K}, f_{\pi}\right)$ to extract the physical values. Our final results are those given in Eq. (2).

\section{$5 \quad$ Scaling laws and related issues}

In this section, we discuss several interesting quantities for the study of the scaling laws predicted by the HQET, and their validity in the range of quark masses between the charmed and the bottom one. We introduce several ratios of decay constants which are useful to get some physical information.

We first consider the scaling law for the decay constants. The results for the coefficients in Eq. (27), as obtained from our fits, are given in Tab. 10. To translate these coefficients into physical units, we have used $a^{-1}\left(m_{K^{*}}\right)$. The leading term from the linear fit, $\Phi_{0}=$

\begin{tabular}{|c||cc|cc|}
\hline \multicolumn{1}{|c||}{ fit } & \multicolumn{2}{c|}{$m_{\ell}=m_{q}$} & \multicolumn{2}{c|}{$m_{\ell}=m_{s}$} \\
parameters & linear & quadratic & linear & quadratic \\
\hline \hline$\Phi_{0}^{P S}\left[\mathrm{GeV}^{3 / 2}\right]$ & $0.48(5)$ & $0.66(11)$ & $0.56(5)$ & $0.74(8)$ \\
$\Phi_{1}^{P S} / \Phi_{0}^{P S}[\mathrm{GeV}]$ & $-0.75(6)$ & $-1.60(22)$ & $-0.83(5)$ & $-1.70(16)$ \\
$\sqrt{\Phi_{2}^{P S} / \Phi_{0}^{P S}}[\mathrm{GeV}]$ & - & $1.03(8)$ & - & $1.08(6)$ \\
\hline$\Phi_{0}^{V}\left[\mathrm{GeV}^{3 / 2}\right]$ & $0.51(7)$ & $0.70(12)$ & $0.61(6)$ & $0.81(10)$ \\
$\Phi_{1}^{V} / \Phi_{0}^{V}[\mathrm{GeV}]$ & $-0.63(9)$ & $-1.62(24)$ & $-0.74(6)$ & $-1.65(17)$ \\
$\sqrt{\Phi_{2}^{V} / \Phi_{0}^{V}}[\mathrm{GeV}]$ & - & $1.09(9)$ & - & $1.08(6)$ \\
\hline
\end{tabular}

Table 10: Fit parameters in physical units for pseudoscalar $(P S)$ and vector $(V)$ heavy-light mesons.

$0.48(5) \mathrm{GeV}^{3 / 2}$, is in good agreement with the findings of previous studies [13, 26, 27, 28].

We also note that this value is compatible with the results of QCD sum rules [29], $\Phi_{0}=$ $(0.4 \div 0.6) \mathrm{GeV}^{3 / 2}$, when the large perturbative QCD corrections are included ${ }^{8}$.

Following the Refs. [21, 28], we now consider the spin scaling relation on the lattice:

$$
U\left(\bar{M}_{H}\right)=\frac{f_{H}}{f_{H^{*}}}=\xi_{0}+\frac{\xi_{1}}{\bar{M}_{H}}+\frac{\xi_{2}}{\bar{M}_{H}^{2}},
$$

\footnotetext{
${ }^{8}$ Without these corrections, the result would be $\Phi_{0}=0.30(5)$ [29].
} 
where $\bar{M}_{H}=\left(3 M_{H^{*}}+M_{H}\right) / 4$ is the spin averaged mass (which we already used in Sec. 3), and $\xi_{0,1,2}$ are parameters which we obtain by fitting our data. From heavy quark symmetry, one expects that $\xi_{0}=1$ (up to logarithmic corrections). For completeness, we tabulated $\bar{M}_{H}$ and $f_{H} / f_{H^{*}}$ in Tab. 11. The results of our fits in physical units, are

\begin{tabular}{|c|cccc|}
\hline 'heavy flavor' & $\kappa_{h 1}$ & $\kappa_{h 2}$ & $\kappa_{h 3}$ & $\kappa_{h 4}$ \\
\hline \hline $\bar{M}_{H}=\left(3 M_{H^{*}}+M_{H}\right) / 4$ & $0.6705(48)$ & $0.7620(53)$ & $0.8486(56)$ & $0.9579(61)$ \\
$\bar{M}_{H_{s}}=\left(3 M_{H_{s}^{*}}+M_{H_{s}}\right) / 4$ & $0.7028(58)$ & $0.7940(58)$ & $0.8802(58)$ & $0.9890(60)$ \\
\hline$U\left(\bar{M}_{H}\right)=f_{H} / f_{H^{*}}$ & $0.851(30)$ & $0.869(29)$ & $0.879(29)$ & $0.888(31)$ \\
$U\left(\bar{M}_{H_{s}}\right)=f_{H_{s}} / f_{H_{s}^{*}}$ & $0.844(22)$ & $0.858(21)$ & $0.867(21)$ & $0.876(22)$ \\
\hline
\end{tabular}

Table 11: Spin averaged masses and ratios of pseudoscalar and vector decay constants. For $\bar{M}_{H}$, the light quark mass $q=u, d$, is understood.

$$
\begin{array}{cl}
\text { (lin.) } \quad \xi_{0}=0.997(68), \quad \xi_{1} / \xi_{0}=-0.23(11) G e V, \\
\text { (quad.) } \quad \xi_{0}=0.89(12), \quad \xi_{1} / \xi_{0}=0.17(49) G e V, \quad \sqrt{\xi_{2} / \xi_{0}}=-0.67(18) G e V,
\end{array}
$$

where the physical values were obtained by using $a^{-1}\left(m_{K^{*}}\right)$. Data points, and extrapolated values, are displayed in Fig. 6. We see that the scaling law is very well satisfied by using the linear fit. The inclusion of the quadratic term, although irrelevant in the directly accessible region of the meson masses, produces large deviation from the expected extrapolated value $\xi_{0}=1$, as $\bar{M}_{H} \rightarrow \infty$. Thus, by using the linear fit, we arrive at

$$
U\left(\bar{M}_{D}\right)=0.860(28), \quad U\left(\bar{M}_{B}\right)=0.933(47),
$$

and

$$
U\left(\bar{M}_{D_{s}}\right)=0.868(21), \quad U\left(\bar{M}_{B_{s}}\right)=0.915(33) .
$$

We end this section by presenting a set of ratios which may be explored in order to extract the physical decay constants by using some measured quantities.

- As it was suggested in Ref. 13], the decay constants can be conveniently presented in terms of $f_{D_{s}}$, which is already measured ${ }^{9}$ :

$$
\frac{f_{B}}{f_{D_{s}}}=0.78 \pm 0.04_{-0}^{+11} ; \quad \frac{f_{B_{s}}}{f_{D_{s}}}=0.88 \pm 0.03_{-0}^{+12}
$$

${ }^{9} f_{D_{s}}^{(\text {exp. })}=254 \pm 31 \mathrm{MeV}$ [14. 


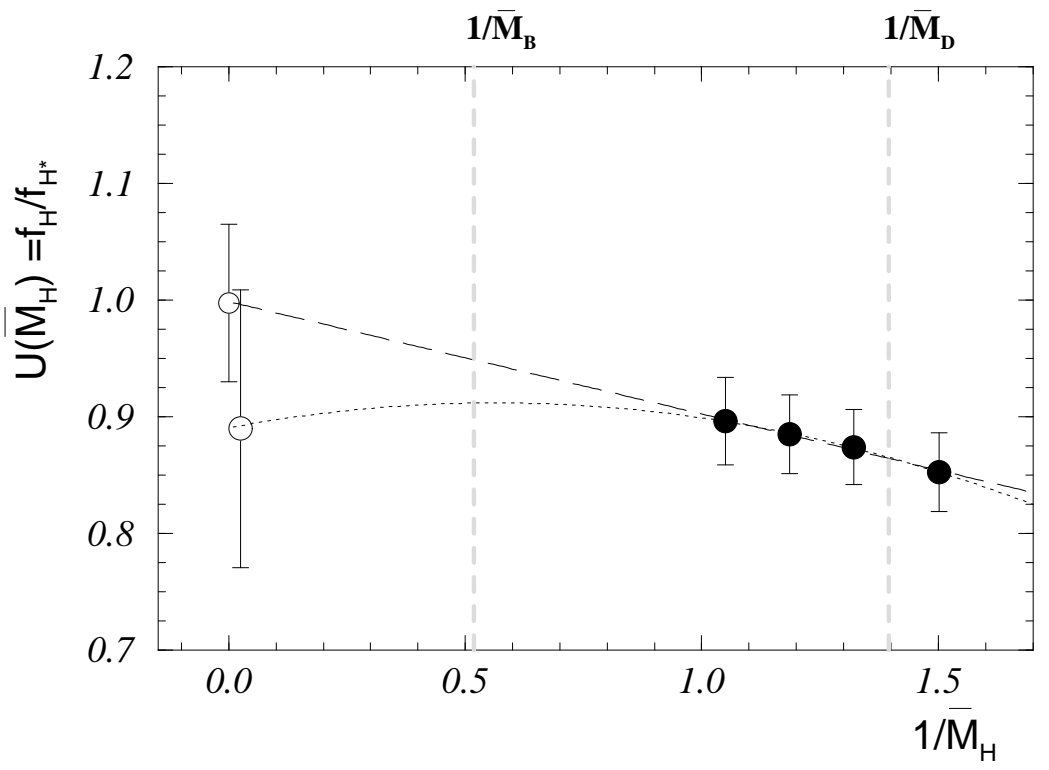

Figure 6: Ratio of the heavy-light decay constants. The linear fit approaches very well the expected asymptotic value $U\left(\bar{M}_{H} \rightarrow \infty\right)=\xi_{0}=1$. The results refer to mesons with the light quark extrapolated to $q=u, d$.

$$
\begin{aligned}
\frac{f_{D^{*}}}{f_{D_{s}}}=1.06 \pm 0.05 ; & \frac{f_{D_{s}^{*}}}{f_{D_{s}}}=1.17 \pm 0.03 ; \\
\frac{f_{B^{*}}}{f_{D_{s}}}=0.85 \pm 0.07_{-0}^{+13} & \frac{f_{B_{s}^{*}}}{f_{D_{s}}}=0.99 \pm 0.05_{-0}^{+14} .
\end{aligned}
$$

The error estimates are obtained in the same way as in Sec. 因. $f_{B} / f_{D_{s}}$ is the value which has been used in Eq. (可), as an alternative way to extract the value for $f_{B}$.

- Other phenomenologically interesting ratios for testing the factorization hypothesis in non-leptonic modes, are (see [30]):

$$
\frac{f_{D_{s}^{*}}}{f_{\rho}}=1.29(14) ; \quad \frac{f_{D_{s}}}{f_{\pi}}=1.66(19) .
$$

- In the quenched approximation, the $S U(3)$-breaking parameter $r_{K}-1 \equiv f_{K} / f_{\pi}-1$, is expected to be smaller than its experimental value. A smaller value of $r_{K}-1$ is predicted by one-loop quenched chiral perturbation theory [31], and is verified in numerous simulations (with either unimproved or improved actions and operators [17]). A similar effect is also expected for $r_{H}=f_{H_{s}} / f_{H}\left(r_{D}=f_{D_{s}} / f_{D}, r_{B}=f_{B_{s}} / f_{B}\right)$ [32]. In this respect (in the hope of reducing the quenching errors), it may be interesting to examine the Grinstein-type double ratio $\mathfrak{R}_{H}=r_{H} / r_{D}$ [33. From our data, we have

$$
\mathfrak{R}_{H_{h_{1}}}=0.995(3), \quad \mathfrak{R}_{H_{h_{2}}}=1.003(4), \quad \mathfrak{R}_{H_{h_{3}}}=1.009(6), \quad \mathfrak{R}_{H_{h_{4}}}=1.014(9) \text {, }
$$


which upon an extrapolation to the B-meson mass, amounts to

$$
\mathfrak{R}_{B}^{(\text {lin })}=1.035(17), \quad \mathfrak{R}_{B}^{(\text {quad })}=1.028(33) .
$$

Using $\mathfrak{R}_{B}^{(\text {lin })}$ and $r_{D}=1.10(2)$ from (1), we have $r_{B}=1.134(34)$, in perfect agreement with the direct determination, given in (2).

- The double ratio can be used to estimate the quenching errors in the predicted values of $r_{D}$ and $r_{B}$. To this purpose, we define

$$
\bar{r}_{H}=\frac{r_{H}}{r_{K}}\left(\frac{f_{K}}{f_{\pi}}\right)^{(\exp .)},
$$

where $r_{H}$ and $r_{K}$, are obtained in the quenched lattice calculation. Using our data $\left(r_{K}=1.12(5)\right.$ [17]) and $\left(f_{K} / f_{\pi}\right)^{(\text {exp. })}=1.22$ [24], we end up with

$$
\begin{aligned}
& \bar{r}_{D}^{\text {(lin.\&quad. })}=1.19(5) \\
& \bar{r}_{B}^{\text {(lin.\&quad. })}=1.23(6),
\end{aligned}
$$

which are $\sim 9 \%$ larger than the results obtained directly and quoted in Eqs. (11) and (2)). If this difference of $9 \%$ is the realistic estimate of the quenching errors, they are much smaller than the pessimistic estimate of Ref. [32], where $\sim 20 \%$ of (quenching) error was predicted. Note that the ratios $\bar{r}_{D}$ and $\bar{r}_{B}$, do not depend on the fit we use (linear or quadratic). A similar game with $f_{B} / f_{D_{s}}$ results in

$$
\frac{f_{B}}{f_{D_{s}}}=0.71 \pm 0.04_{-0}^{+10}
$$

which gives $f_{B}=(180 \pm 26 \text { (exp. })_{-10}^{+29}$ (theo. $\left.)\right) \mathrm{MeV}$, where we accounted for the experimental value for $f_{D_{s}}^{(e x p .)}$. This result agrees with the value we reported in (2). 


\section{Conclusion}

We have analyzed masses and decay constants of heavy-light pseudoscalar and vector mesons, using the non-perturbatively improved action and currents. Particular attention has been paid to the errors coming from the extrapolation in the light and heavy quark masses.

We find that the hyperfine splitting is definitely below the experimental value, in spite of the improved action.

The values predicted for the decay constants of $D$ mesons are extremely stable against variations of the fitting procedure, inclusion of KLM factors etc. Thus we believe that the main error on these quantities is the quenching error.

On the contrary, we find larger uncertainties for the $B$-meson decay constants, mainly due to the amplification of discretization effects when extrapolating to the $b$-quark mass, and to the uncertainty in the extrapolation procedure. In spite of these uncertainties, and of the fact that our results are obtained at a single value of the lattice spacing, we believe a value of $f_{B}$ much lower than $170 \mathrm{MeV}$ rather unlikely. Indeed, for $\beta \geq 6.0$, with Wilson-like fermions at fixed lattice spacing, almost all lattice calculations give $f_{B}$ larger than $160 \mathrm{MeV}$. This value has been quoted as the "world average" obtained in Ref. [34], after combining results obtained with propagating quarks, with those obtained using some effective theory, as NRQCD [35], or the FNAL action [36]. Low values of $f_{B}$ with propagating quarks are obtained only after extrapolating in $a$ to the continuum limit [13], 34], with procedures which we believe are questionable (for example by including data at low values of $\beta$, i.e. too close to the strong coupling regime). Our results, which should have smaller discretization errors than other calculations at fixed lattice spacing, confirm a value of $f_{B}$ (in the quenched approximation) larger than $170 \mathrm{MeV}$. A (rather) indirect evidence that a larger value of $f_{B}$ is preferred can be obtained by combining $f_{B} / f_{D_{s}}$ from the lattice with the experimental value of $f_{D_{s}}$. This gives $f_{B} \simeq 180 \div 190 \mathrm{MeV}$, with an error of about $40 \mathrm{MeV}$. Finally, we used the Grinstein double-ratio method, in order to try to reduce the quenching errors for (ratios of) decay constants.

\section{Acknowledgements}

We thank L. Lellouch and S. Sharpe for interesting discussions on the subject of this paper. We also thank F. Parodi for informing us on the updated value of $f_{D_{s}}^{(e x p .)}$. D.B. acknowledges the support of "La Fondation des Treilles". V.L. and G.M. acknowledge the M.U.R.S.T. and the INFN for partial support. G.M. thanks the CERN TH Division for hospitality during the completion of this work. 


\section{References}

[1] B. Sheikloleslami and R. Wohlert, Nucl. Phys. B259 (1985) 572,

G. Heatlie et al., Nucl. Phys. B352 (1991) 266.

[2] M. Lüscher, S. Sint, R. Sommer, P. Weisz, Nucl. Phys. B478 (1996) 365.

[3] M. Lüscher, S. Sint, R. Sommer, P. Weisz, U. Wolff, Nucl. Phys. B491 (1997) 323.

[4] M. Lüscher, S. Sint, R. Sommer, H. Wittig, Nucl. Phys. B491 (1997) 344.

[5] M. Lüscher, Summer School in Theoretical Physics, Session 68, Les Houches 97, hep-lat/9802029.

[6] M. Bochicchio et al., Nucl. Phys. B262 (1985) 331.

[7] M. Crisafulli, V. Lubicz and A. Vladikas, Eur.Phys.J.C4 (1998) 145.

[8] M. Guagnelli, R. Sommer, Nucl. Phys. B63 (Proc. Suppl.) (1998), 886, R. Sommer, Nucl. Phys. 60A(Proc. Suppl.) (1998), 279.

[9] G. Martinelli, G.C. Rossi, C.T. Sachrajda, S. Sharpe, M. Talevi, M. Testa, Phys. Lett. B411 (1997) 141.

[10] T. Bhattacharya, S. Chandrasekharan, R. Gupta, W. Lee, S. Sharpe, in preparation; see also hep-lat/9810018 (Talk presented at the International Symposium 'Lattice 98', Boulder, July 13-18, 1998).

[11] S. Sint, P. Weisz, Nucl. Phys. B502 (1997) 251 (and references therein).

[12] M. Neubert, B. Stech, in "Heavy Flavours II", edited by A.J. Buras and M. Lindner (World Scientific, Singapore), hep-lat/9705292,

for recent application see M. Ciuchini, R. Contino, E. Franco, G. Martinelli, hep-ph/9810271.

[13] C. Bernard et al. (MILC - Coll.), hep-lat/9806412.

[14] F. Parodi, P. Roudeau, A. Stocchi, hep-ph/9802289.

[15] H. Wittig, Int. J. Mod. Phys. A12 (1997) 4477.

[16] J.M. Flynn, C.T. Sachrajda, In A.J. Buras, M. Lindner, "Heavy flavours II" pp.402 (World Scientific, Singapore), hep-lat/9710057.

[17] D. Becirevic, Ph. Boucaud, L. Giusti, J.P. Leroy, V. Lubicz, G. Martinelli, F. Mescia, F. Rapuano, hep-lat/9809129.

[18] D. Becirevic, Ph. Boucaud, J.P. Leroy, V. Lubicz, G. Martinelli, F. Mescia, hep-lat/9807046.

[19] C.R. Allton, V. Gimenez, L. Giusti, F. Rapuano, Nucl. Phys. B489 (1997) 427.

[20] S. Capitani et al., Talk given at " $2^{\text {nd }}$ Topical Workshop on Deep Inelastic Scattering off Polarized Targets", Zeuthen, Germany, hep-lat/9711007. 
[21] M. Bochicchio, G. Martinelli, C.R. Allton, C.T. Sachrajda, D.B. Carpenter, Nucl. Phys. B372 (1992) 403,

A. Abada et al., Nucl. Phys. B376 (1992) 172.

[22] D. Becirevic et al. (APE - Coll.), presented at the International Symposium 'Lattice 98', Boulder, July 13-18, 1998, hep-lat/9809187.

[23] D. Richards et al. (UKQCD - Coll.), hep-lat/9809074

A. Cucchieri, T. Mendes, R. Petronzio (APETOV - Coll.), hep-lat/9810028 both presented at the Int. Symposium 'Lattice 98', Boulder, July 13-18, 1998.

[24] C. Caso et al. (Particle Data Group), Eur. Phys. J. C3 (1998) 1.

[25] G.P. Lepage and P.B. Mackenzie, Phys. Rev. D48 (1993), 2250, A.X. El-Khadra, A.S. Kronfeld and P.B. Mackenzie, Phys. Rev. D55 (1994) 3933, C.W. Bernard, Nucl. Phys. B34 (Proc. Suppl.) (1994), 47.

[26] C.R. Allton et al. (APE - Coll.), Phys. Lett. B405 (1997) 133.

[27] S. Aoki et al. (JLQCD - Coll.), Nucl. Phys. B53(Proc. Suppl.) (1997), 355.

[28] R.M. Baxter et al. (UKQCD - Coll.), Phys. Rev. D49 (1994) 1594.

[29] D.J. Broadhurst, A.G. Grozin, Phys. Lett. B274 (1992) 421, M. Neubert, Phys. Rev. D46 (1992) 1076, E. Bagan et al., Phys. Lett. B278 (1992) 457.

[30] M. Neubert, Nucl. Phys. B64 (Proc. Suppl.) (1998), 474.

[31] S. Sharpe, Nucl. Phys. B17 (Proc. Suppl.) (1990), 146, ibid B53 (1997), 181, C. Bernard, M. Golterman, Phys. Rev. D46 (1992) 853.

[32] S. Sharpe, Y. Zhang, Phys. Rev. D53 (1996) 5125.

[33] C.G. Boyd, B. Grinstein,Nucl. Phys. B442 (1995) 205.

[34] T. Draper, rapporteur talk given at International Symposium 'Lattice 98', Boulder, July 13-18, 1998, hep-lat/9810065.

[35] A. Ali Khan, T. Bhattacharya, S. Collins, C.T.H. Davies, R. Gupta, C. Morningstar, J. Shigemitsu, J. Sloan, Phys. Lett B427 (1998) 132.

[36] A.X. El-Khadra, A.S. Kronfeld, P.B. Mackenzie, S.M. Ryan, J.N. Simone, Phys. Rev. D58 (1998) 014506. 